\title{
Relación entre la depresión, la ansiedad y los síntomas psicosomáticos en una muestra de estudiantes universitarios del norte de México
}

\author{
Mónica Teresa González Ramírez, ${ }^{1}$ René Landero Hernández ${ }^{1}$ \\ y Javier García-Campayo ${ }^{2}$
}

Forma de citar

González Ramírez MT, Landero Hernández R, García-Campayo J. Relación entre la depresión, la ansiedad y los síntomas psicosomáticos en una muestra de estudiantes universitarios del norte de México. Rev Panam Salud Publica. 2009;25(2):141-5.

RESUMEN Objetivo. Determinar la relación existente entre la ansiedad, la depresión y los síntomas psicosomáticos en una muestra de estudiantes universitarios del norte de México.

Métodos. Se realizó un estudio exploratorio a partir de cuestionarios de autoinforme aplicados a una muestra de conveniencia de 506 estudiantes de psicología de dos universidades de Monterrey, estado de Nuevo León, México. Para evaluar los síntomas psicosomáticos se utilizó el cuestionario sobre la salud del paciente, para la depresión se empleó el inventario de Beck y la ansiedad se evaluó mediante la escala social para adolescentes. Se utilizó la correlación de Spearman para determinar el nivel de significación de la relación entre las variables y la prueba de Kruskal-Wallis para comparar los niveles de ansiedad y depresión en los grupos de estudiantes conformados según la intensidad de los síntomas psicosomáticos.

Resultados. De los participantes, $129(25,5 \%)$ presentaron síntomas psicosomáticos de intensidad media o alta, solo 4 (0,8\%) manifestaron depresión intensa y únicamente $2(0,4 \%)$ estudiantes presentaron niveles de ansiedad por encima del $75 \%$ de la puntuación máxima de la escala. La intensidad de los síntomas psicosomáticos aumentó a la par que aumentaban los niveles de ansiedad y depresión. Los sintomas psicosomáticos más frecuentes y que causaban mayor preocupación en la muestra fueron: dolores de cabeza, menstruales y de espalda, así como sentirse cansado y dificultades para dormir.

Conclusiones. Se confirmó la relación directa entre la intensidad de los sintomas somáticos, la depresión y la ansiedad. Se recomienda incluir en los programas de prevención o tratamiento de cualquiera de estos tres trastornos a los otros dos. Se deben implementar programas específicos para los jóvenes universitarios.

Palabras clave Ansiedad, depresión, trastornos psicosomáticos, estudiantes, México.

Facultad de Psicología, Universidad Autónoma de Nuevo León, Monterrey, Nuevo León, México. La correspondencia se debe dirigir a Mónica T. González Ramírez, Facultad de Psicología, Universidad Autónoma de Nuevo León, Av. Mutualismo No. 110, Colonia Mitras Centro, Monterrey, Nuevo León 64460, México. Correos electrónicos: monygzz77@yahoo.com y monica.gonzalezrz@ uanl.edu.mx
Cuando una persona siente dolor o algún otro síntoma somático tiende a atribuirlo a alguna enfermedad, sin embargo, de las molestias que padecemos

2 Hospital Universitario Miguel Servet y Universidad de Zaragoza, Zaragoza, España. solo $5 \%$ se debe a enfermedades, mientras 70\% está relacionada con causas naturales o ambientales y el $25 \%$ restante obedece a causas psicológicas (1). Farré y Fullana (2) confirmaron que hasta $80 \%$ de las personas sanas pueden haber experimentado en su vida molestias de 
hasta una semana sin una causa clínica. Las molestias físicas sin explicación médica corresponden a los denominados trastornos de somatización.

Actualmente es frecuente que los trastornos de somatización y los psicosomáticos se consideren lo mismo. Sin embargo, ambos conceptos hacen referencia a la relación entre lo psíquico y lo físico desde diferentes puntos de vista: en los trastornos psicosomáticos (también llamados trastornos psicofisiológicos) existe una dolencia física real a la que factores psicológicos pueden vincularse - ya sea en su aparición, mantenimiento o agravamiento-, mientras en los trastornos de somatización el diagnóstico médico no proporciona ninguna justificación de los síntomas (3). Para los efectos del presente trabajo, el término síntoma psicosomático se utiliza cuando existe una dolencia física - como dolor de estómago, espalda, brazos, piernas, cabeza o pecho, vértigo, falta de aire, estreñimiento, indigestión, entre otros- sin que se alcance un diagnóstico médico que explique suficientemente esos síntomas (4).

Los síntomas psicosomáticos y los trastornos de somatización son frecuentes. En un estudio patrocinado por la Organización Mundial de la Salud (5) con el objetivo de examinar la prevalencia de estos síntomas en 5438 personas de 14 países (Alemania, Brasil, Chile, China, Estados Unidos de América, Francia, Grecia, India, Italia, Japón, Nigeria, Países Bajos, Reino Unido y Turquía) se encontraron valores desde 7,6\% (Nigeria) hasta 36,8\% (Chile), con una media de $19,7 \%$. En Alemania, en un estudio epidemiológico prospectivo con una muestra de 2548 adolescentes y adultos jóvenes se encontró que la tasa de incidencia era de 25,7\% (6). En España, 34,5\% de una muestra representativa de 1559 pacientes atendidos en instituciones de salud primarias presentaba trastornos de somatización (7). El porcentaje de síntomas psicosomáticos de intensidad media o alta encontrado en una muestra de 234 estudiantes universitarios de México fue de 21,8\% (8).

Debido a que diversos factores se han asociado con el origen de los síntomas y los trastornos psicosomáticos, su estudio se debe basar en investigaciones multifactoriales y multidisciplinarias (9). La presencia de depresión y ansiedad son dos de las variables asociadas más frecuentemente con los síntomas psicosomáticos, ya que los pacientes con síntomas médicamente inexplicables manifiestan más síntomas de depresión y ansiedad que los pacientes con síntomas con explicación médica (10). Varios investigadores han encontrado asociaciones significativas entre los trastornos de somatización por una parte y la ansiedad $(5,11)$ y la depresión, por la otra $(6,11)$.

Sin embargo, las investigaciones publicadas hasta el momento no aclaran suficientemente en qué grado pueden estar presentes los síntomas psicosomáticos en personas consideradas sanas, sin diagnóstico previo de trastornos de depresión, ansiedad o somatización. El objetivo del presente trabajo fue determinar la relación existente entre la ansiedad, la depresión y los síntomas psicosomáticos en una muestra de estudiantes universitarios del norte de México.

\section{MATERIALES Y MÉTODOS}

Se realizó un estudio exploratorio basado en cuestionarios de autoinforme. En el estudio participaron 506 estudiantes de psicología de dos universidades ubicadas en la ciudad de Monterrey, capital del estado de Nuevo León, México.

La muestra, tomada por conveniencia, quedó compuesta por estudiantes de todos los semestres de las clases impartidas por colaboradores de la investigación: $76(15,0 \%)$ estudiantes de primer semestre, $20(4,0 \%)$ de segundo semestre, $43(8,5 \%)$ de tercer semestre, $31(6,1 \%)$ de cuarto semestre, $59(11,7 \%)$ de quinto semestre, $31(6,1 \%)$ de sexto semestre; 91 $(18,0 \%)$ de séptimo semestre, 48 (9,5\%) de octavo semestre, $70(13,8 \%)$ de noveno semestre y $28(5,5 \%)$ de décimo semestre; 9 (1,8\%) estudiantes no informaron su semestre de estudio. En una de las universidades, el sistema de calificación es por créditos y flexible, mientras que en la otra el plan de estudios es rígido, por lo que los semestres de estudio no indican que el historial académico de los estudiantes de esas universidades sean equivalentes.

Los estudiantes aceptaron participar voluntariamente en esta investigación después de recibir información sobre su propósito y el respeto a los principios de confidencialidad. Los participantes respondieron los cuestionarios en las instalaciones de sus facultades 10 días antes de los exámenes de mitad de curso correspondientes al segundo semestre (período académico agosto-diciembre de 2005); la fecha de los exámenes varió de una universidad a otra.
Para la medición de los síntomas psicosomáticos se utilizó el cuestionario sobre la salud del paciente (Patient Health Questionnaire, PHQ) (12). Este instrumento, que puede emplearse en población general, da una medida de la intensidad de los síntomas y permite identificar a los pacientes que podrían desarrollar síntomas clínicos a partir de estos trastornos.

El PHQ está compuesto por 15 preguntas con respuestas de tipo Likert con tres opciones de respuesta. Trece preguntas exploraron los síntomas somáticos y el grado de preocupación que producían en los entrevistados (nada: 0 punto; un poco: 1 punto; mucho; 2 puntos) y dos abordaron la preocupación de los entrevistados en las últimas dos semanas por síntomas psicosomáticos relacionados con la depresión (nunca: 0 punto; algunos días: 1 punto; más de la mitad de los días: 2 puntos) (12). A partir de la puntuación alcanzada, los entrevistados se clasificaron según las cuatro categorías de intensidad propuestas por los autores del PHQ: mínima: 0-4; baja: 5-9; media: 10-14; y alta: 15-30. La consistencia interna del instrumento en su versión original en inglés, medida por el coeficiente $\alpha$ de Cronbach, fue de 0,80 (12), mientras que en un estudio donde se analizaron sus propiedades psicométricas en una muestra con hispanos y no hispanos $(n=172)$ con altos niveles de síntomas médicamente inexplicables, la consistencia interna se mantuvo elevada en ambos grupos $(\alpha=0,79)(13)$. También se ha informado una elevada consistencia interna en población mexicana $(\alpha=0,77)(8)$.

Para evaluar la depresión se utilizó el inventario de Beck (14), que consta de 21 preguntas sobre los síntomas depresivos con cuatro opciones de respuesta (de 0 a 3 puntos) según la intensidad del síntoma. La puntuación total (entre $0 \mathrm{y}$ 63 puntos) se obtuvo por la suma de las puntuaciones de cada síntoma explorado. La consistencia interna de este instrumento en varios estudios ha sido satisfactoria ( $\alpha$ de Cronbach de 0,76 a 0,95 ) (15). Los participantes se clasificaron según su nivel de depresión en cuatro categorías: sin depresión: de 0 a 9 puntos; depresión leve: de 10 a 18; depresión moderada: de 19 a 29; y depresión intensa: 30 puntos o más (15).

La ansiedad se evaluó mediante la escala social para adolescentes (16), desarrollada a partir de la escala revisada 
para la ansiedad social en niños, en la que únicamente se modificó el término "niño" por "compañero", "otras personas" o "gente", y el término "jugar con otros" por "hacer cosas con otros". Con esta escala se evaluaron las respuestas sobre la ansiedad social de los adolescentes en el contexto de sus relaciones interpersonales (17). Su consistencia interna en población de habla hispana fue de 0,91 (18). La puntuación total en esta escala fue de 0 a 72 puntos.

Se realizaron análisis descriptivos para todas las variables. Debido a que los datos no mostraron una distribución normal según la prueba de KolmogorovSmirnov $(P=0,001$ en las tres variables del estudio) se utilizó la correlación de Spearman para determinar el nivel de significación de la relación entre las variables. Al no cumplirse los supuestos de normalidad y homocedasticidad de las varianzas, se utilizó la prueba de Kruskal-Wallis para comparar los niveles de ansiedad y depresión en los grupos de estudiantes conformados según la intensidad de los síntomas psicosomáticos.

A los estudiantes que lo solicitaron y que no hubieran respondido de forma anónima los cuestionarios se les entregaron los resultados individuales con la puntuación obtenida en cada variable y su comparación con respecto a la muestra total y a los criterios de clasificación de los instrumentos empleados. Se recomendó psicoterapia o buscar cursos o talleres para el control de la ansiedad, los síntomas depresivos o los síntomas psicosomáticos, según el caso, a los estudiantes que presentaron puntuaciones superiores tanto a la media, como a la mediana de la muestra en los cuestionarios utilizados.

\section{RESULTADOS}

De los 506 estudiantes participantes, $97(19,2 \%)$ eran hombres y $409(80,8 \%)$ eran mujeres; la edad promedio fue de 20,71 \pm 3,39 años (mediana: 20 años). Del total de participantes, $483(95,3 \%)$ eran solteros, $19(3,9 \%)$ estaban casados, $1(0,2 \%)$ era viudo y $1(0,2 \%)$ estaba divorciado; $2(0,4 \%)$ eran madres solteras.

$\mathrm{Al}$ analizar las medidas de tendencia central, se encontró que la media y la mediana de las puntuaciones de los síntomas psicosomáticos fueron muy bajas en comparación con las puntuaciones máximas posibles del PHQ (cuadro 1). En general, $129(25,5 \%)$ participantes pre-

CUADRO 1. Comportamiento de las variables del estudio en la muestra de estudiantes universitarios analizada $(n=506)$, México, 2005

\begin{tabular}{|c|c|c|c|c|}
\hline \multirow[b]{2}{*}{ Variable } & \multirow[b]{2}{*}{ Escala } & \multicolumn{2}{|c|}{ Puntuación de la muestra } & \multirow{2}{*}{$\begin{array}{l}\text { Desviación } \\
\text { estándar }\end{array}$} \\
\hline & & Mediana & Media & \\
\hline Síntomas psicosomáticos & 0 a 30 & 6,00 & 7,03 & 4,02 \\
\hline Depresión & 0 a 63 & 6,00 & 6,73 & 5,52 \\
\hline Ansiedad & 0 a 72 & 15,00 & 17,02 & 11,43 \\
\hline
\end{tabular}

CUADRO 2. Frecuencia de síntomas psicosomáticos, depresión y ansiedad en la muestra de estudiantes universitarios analizada, según la clasificación de intensidad de las escalas empleadas, México, 2005

\begin{tabular}{lrr}
\hline Variable/intensidad & No. & $\%$ \\
\hline Síntomas psicosomáticos & & \\
Mínima & 147 & 29,0 \\
Baja & 230 & 45,5 \\
Media & 100 & 19,8 \\
Alta & 29 & 5,7 \\
Depresión & & \\
Sin depresión & 381 & 75,3 \\
Leve & 107 & 21,1 \\
Moderada & 14 & 2,8 \\
Intensa & 4 & 0,8 \\
Ansiedad & & \\
Grupo 1 (0-18 puntos) & 316 & 62,5 \\
Grupo 2 (19-36 puntos) & 155 & 30,6 \\
Grupo 3 (37-54 puntos) & 33 & 6,5 \\
Grupo 4 (55 puntos o más) & 2 & 0,4 \\
\hline a La clasificación de la ansiedad en cuatro grupos se realizó de manera arbitraria a par-
\end{tabular}

tir de la puntuación de la escala ( 0 a 72). Esta clasificación no tiene valor diagnóstico.

CUADRO 3. Comparación de los niveles de depresión y ansiedad en la muestra de estudiantes universitarios analizada, según la intensidad de los síntomas psicosomáticos, México, 2005

\begin{tabular}{|c|c|c|c|c|c|c|c|c|}
\hline \multirow{2}{*}{$\begin{array}{l}\text { Intensidad de } \\
\text { los síntomas } \\
\text { psicosomáticos }\end{array}$} & \multicolumn{4}{|c|}{ Nivel de depresión } & \multicolumn{4}{|c|}{ Nivel de ansiedad } \\
\hline & Media & $\mathrm{DE}^{\mathrm{a}}$ & $\chi^{2 b}$ & $P^{\mathrm{b}}$ & Media & DE & $\chi^{2 b}$ & $P^{\mathrm{b}}$ \\
\hline Mínima & 3,81 & 3,62 & \multirow{4}{*}{95,472} & \multirow{4}{*}{0,001} & 13,95 & 10,01 & \multirow{4}{*}{21,999} & \multirow{4}{*}{0,001} \\
\hline Baja & 6,85 & 4,71 & & & 16,67 & 10,24 & & \\
\hline Media & 9,12 & 6,48 & & & 20,85 & 12,96 & & \\
\hline Alta & 12,28 & 7,34 & & & 22,17 & 15,84 & & \\
\hline
\end{tabular}

a DE: desviación estándar.

b Según la prueba de Kuskal-Wallis (grados de libertad: 3 ).

sentaban síntomas psicosomáticos de intensidad media o alta. En cuanto al diagnóstico de la depresión a partir de los puntos de corte establecidos, se encontró que $488(96,4 \%)$ de los participantes no presentaban depresión o esta era leve y solo $4(0,8 \%)$ participantes presentaron depresión intensa (cuadro 2).

En cuanto a la ansiedad mostrada por los participantes se observó que la mitad de los estudiantes de la muestra presentaron un nivel de ansiedad social por debajo de 15 puntos (cuadro 1). Como la escala de ansiedad empleada no cuenta con puntos de corte de valor diagnóstico para analizar la intensidad de la ansie- dad, para este trabajo se estableció una clasificación arbitraria de la ansiedad a partir de la puntuación máxima posible de la escala empleada. De acuerdo con esta clasificación, $316(62,5 \%)$ de los participantes presentaban puntuaciones de ansiedad por debajo del $25 \%$ del máximo de la escala y únicamente $2(0,4 \%)$ estudiantes presentaron puntuaciones por encima del $75 \%$ (cuadro 2).

Los valores medios de depresión y ansiedad se incrementaron al aumentar la intensidad de los síntomas psicosomáticos (cuadro 3), con diferencias significativas entre los niveles de depresión y ansiedad que padecían los participantes 
CUADRO 4. Frecuencia de los principales síntomas psicosomáticos en la muestra de estudiantes universitarios analizada, México, 2005

\begin{tabular}{|c|c|c|c|c|c|c|}
\hline \multirow[b]{2}{*}{ Síntomas psicosomáticos } & \multicolumn{2}{|c|}{ No preocupado } & \multicolumn{2}{|c|}{ Preocupado } & \multicolumn{2}{|c|}{ Muy preocupado } \\
\hline & No. & $\%$ & No. & $\%$ & No. & $\%$ \\
\hline Dolores de cabeza & 206 & 40,7 & 220 & 43,5 & 80 & 15,8 \\
\hline Dolores menstruales ${ }^{a}$ & 176 & 43,0 & 170 & 41,6 & 63 & 15,4 \\
\hline Dolor de espalda & 219 & 43,3 & 220 & 43,5 & 67 & 13,2 \\
\hline Estreñimiento, movimientos intestinales o diarrea & 265 & 52,4 & 182 & 36,0 & 59 & 11,7 \\
\hline Dolor en brazos, piernas, articulaciones & 290 & 57,3 & 183 & 36,2 & 33 & 6,5 \\
\hline Nauseas, gases o indigestión & 349 & 69,0 & 126 & 24,9 & 31 & 6,1 \\
\hline Dolor de estómago & 263 & 52,0 & 213 & 42,1 & 30 & 5,9 \\
\hline Palpitación o taquicardias & 415 & 82,0 & 67 & 13,2 & 24 & 4,7 \\
\hline Sensación de falta de aire & 375 & 74,1 & 107 & 21,1 & 24 & 4,7 \\
\hline Vértigo & 385 & 76,1 & 98 & 19,4 & 23 & 4,5 \\
\hline Dolor de pecho & 416 & 82,2 & 71 & 14,0 & 19 & 3,8 \\
\hline Dolor o problemas durante las relaciones sexuales & 472 & 93,3 & 25 & 4,9 & 9 & 1,8 \\
\hline Síncopes o desvanecimientos & 477 & 94,3 & 22 & 4,3 & 7 & 1,4 \\
\hline
\end{tabular}

a Calculado para 409 mujeres.

CUADRO 5. Frecuencia de síntomas psicosomáticos relacionados con la depresión en la muestra de estudiantes universitarios analizada, México, 2005

\begin{tabular}{|c|c|c|c|c|c|c|}
\hline \multirow[b]{2}{*}{ Síntomas psicosomáticos } & \multicolumn{2}{|c|}{ Nunca } & \multicolumn{2}{|c|}{ Algunos días } & \multicolumn{2}{|c|}{$\begin{array}{c}\text { Más de la mitads } \\
\text { de los días }\end{array}$} \\
\hline & No. & $\%$ & No. & $\%$ & No. & $\%$ \\
\hline Cansado o con poca energía & 68 & 13,4 & 295 & 58,3 & 143 & 28,3 \\
\hline Dificultad para dormir & 250 & 49,4 & 186 & 36,8 & 70 & 13,8 \\
\hline
\end{tabular}

clasificados en las cuatro categorías de síntomas psicosomáticos.

Los coeficientes de correlación de Spearman obtenidos entre los síntomas psicosomáticos y la depresión $\left(r_{\mathrm{s}}=0,446\right.$; $P=0,001)$, los síntomas psicosomáticos y la ansiedad $\left(r_{\mathrm{s}}=0,211 ; P=0,001\right)$ y la ansiedad y la depresión $\left(r_{\mathrm{s}}=0,315\right.$; $P=0,001)$ confirmaron la tendencia a aumentar la intensidad de los síntomas psicosomáticos a la par que aumentaban los niveles de ansiedad y depresión.

Los síntomas psicosomáticos más frecuentes y que causaban mayor preocupación en la muestra fueron: dolores de cabeza, menstruales y de espalda, así como sentirse cansado y dificultades para dormir (cuadros 4 y 5). De los trastornos explorados, los de menor preocupación para los entrevistados fueron el dolor o los problemas durante las relaciones sexuales y los síncopes o desvanecimientos.

\section{DISCUSIÓN}

Se ha demostrado la relación que existe entre los síntomas psicosomáticos, por una parte, y la ansiedad y la depresión, por la otra, en personas con diferentes enfermedades (10), pero son po$\cos$ los estudios de este tipo realizados en personas sanas, lo que resalta la importancia de este trabajo.

La presencia de síntomas psicosomáticos en $25,5 \%$ de la muestra estudiada es ligeramente superior a la observada en un estudio anterior realizado también en estudiantes universitarios (8). La mayor frecuencia de estudiantes de esta muestra con síntomas de intensidad media o alta podría deberse a la elevada proporción de mujeres en la muestra - ya que en ellas son más frecuentes estos síntomas (19)_y a haber aplicado los instrumentos de evaluación días antes del período de exámenes, cuando el estrés puede manifestarse como síntomas psicosomáticos.

Se ha documentado la comorbilidad de los trastornos de somatización con otros, como la depresión mayor o la ansiedad (55\% y $34 \%$ de los pacientes, respectivamente) (20). Además, se ha propuesto que la ansiedad y la depresión, entre otras variables, pueden ser factores de predicción de nuevos episodios de trastornos de somatización (6). A pesar de que los participantes en este estudio se consideraban sanos y pocos de ellos presentaron niveles elevados de depresión o ansiedad, se confirmó la relación entre la depresión y los síntomas psicosomáticos, entre estos y la ansiedad y entre la ansiedad y la depresión. Esto es congruente con las asociaciones ya informadas entre estos tres trastornos en diferentes poblaciones $(6,10,11,20)$. Se debe resaltar que los instrumentos utilizados para evaluar la depresión y la ansiedad se centran principalmente en síntomas cognitivos, lo que evita la circularidad en las mediciones de las variables. Por ejemplo, en el caso de la ansiedad, las preguntas están orientadas a la ansiedad social más que a los síntomas somáticos de trastornos de ansiedad.

Se deben tomar en cuenta los síntomas somáticos más frecuentes encontrados en este grupo, en particular sentirse cansado o con poca energía, tener dificultades para dormir y padecer dolores de cabeza, menstruales y de espalda. Investigar estos síntomas en la población estudiantil puede ser de gran importancia, ya que no solo pueden afectar las calificaciones y las actividades propias como estudiantes, sino también influir negativamente en el rendimiento académico en el sentido más amplio (asistencia a clases, motivación hacia el estudio, actitud hacia el sistema de enseñanza, satisfacción, sensación de logro, ajuste, entre otros) (21).

$\mathrm{Al}$ analizar los resultados del presente trabajo se deben tomar en cuenta algunas limitaciones. En primer lugar, el empleo de una muestra no representativa impide extrapolar los resultados al universo de estudiantes analizado. No se llevó un registro del número de estudiantes de cada clase o de cuántos rechazaron participar en el estudio. Sin embargo, al no tratarse de un muestreo aleatorio, esto último no debe afectar a las conclusiones.

A pesar de esas limitaciones, este estudio pone de manifiesto la importancia de 
considerar la asociación entre sí de los tres trastornos analizados (la ansiedad, la depresión y los síntomas psicosomáticos) en una población joven considerada sana. El hecho de confirmar que los participantes con síntomas somáticos de mayor intensidad también presentaban mayor intensidad en los síntomas de de-

1. García-Campayo J. Usted no tiene nada. La somatización. Barcelona: Océano; 1999.

2. Farré F, Fullana M. Recomendaciones terapéuticas en terapia cognitivo-conductual. Barcelona: Ars Médica; 2005.

3. López J, Belloch A. La somatización como síntoma y como síndrome: una revisión del trastorno de somatización. Rev Psicopatol Psicol Clin. 2002;7(2):73-93.

4. González M, Landero R. Síntomas psicosomáticos y teoría transaccional del estrés. Ansiedad Estrés. 2006;12(1):45-61.

5. Gureje O, Simon G, Ustun T, Goldberg D. Somatization in cross-cultural perspective: a World Health Organization study in primary care. Am J Psychiatry. 1997;154(7):989-95.

6. Lieb R, Zimmermann P, Friss R, Hofler M, Tholen S, Wittchen $\mathrm{H}$. The natural course of DSM-IV somatoform disorders and syndromes among adolescents and young adults: a prospective-longitudinal community study. Eur Psychiatry. 2002;17(6):321-31.

7. Lobo A, García-Campayo J, Campos R, Mar$\cos$ G, Pérez-Echeverría MJ, Working Group for the Study of the Psychiatric and Psychosomatic Morbidity in Zaragoza. Somatisation in primary care in Spain. I: estimates of prevalence and clinical characteristics. Br J Psychiatry. 1996;168:344-8.

8. González M, Landero R. Síntomas psicosomáticos y estrés en estudiantes de Psicología. Rev Psicol Social. 2006;21(2):141-52. presión y ansiedad demuestra la necesidad de incluir en los programas de prevención o tratamiento de cualquiera de estos tres trastornos a los otros dos.

Es necesario diseñar investigaciones dirigidas a determinar las repercusiones que trastornos como la ansiedad, la depresión y los síntomas psicosomáticos

\section{REFERENCIAS}

9. Sandín B, Chorot P, Santed M, Jiménez M. Trastornos psicosomáticos. En: Belloch $\mathrm{A}$, Sandín B, Ramos F, eds. Manual de psicopatología. Vol. 2. Madrid: McGraw-Hill; 1995. Pp. 402-69.

10. Kooiman C, Bolk J, Brand R, Trijsburg R, Rooijmans $\mathrm{H}$. Is alexithymia a risk factor for unexplained physical symptoms in general medical outpatients? Psychosom Med. 2000; 62:768-78.

11. Lipsane T, Saarijävi S, Lauerma H. Exploring the relations between depression, somatization, dissociation and alexithymia-overlapping or independent constructs? Psychopathol. 2004; 37(4):200-6.

12. Kroenke K, Spitzer RL, Williams J. The PHQ15: validity of a new measure for evaluating the severity of somatic symptoms. Psychosom Med. 2002;64:258-66.

13. Interian A, Allen L, Gara M, Escobar J, DíazMartínez A. Somatic complaints in primary care: further examining the validity of the Patient Health Questionnaire (PHQ-15). Psychosomatics. 2002;47(5):392-8.

14. Beck A, Ward C, Mendelson M, Mock J, Erbauhg J. An inventory for measuring depression. Arch Gen Psychiatr. 1961;4:561-71.

15. Beck A, Steer R, Garbin M. Psychometric properties of the Beck Depression Inventory. Twenty-five years of evaluation. Clin Psychol Rev. 1988;8:77-100. producen en el rendimiento académico y la formación profesional de los universitarios. Se deben implementar programas de prevención y atención para los jóvenes universitarios, en los que se apliquen técnicas para el control simultáneo de la depresión, la ansiedad y los síntomas psicosomáticos.
16. La Greca AM, López N. Social anxiety among adolescents: linkages with peer relations and friendships. J Abnorm Child Psychol. 1998;26(2): 83-94.

17. Inglés $C$, Méndez X, Hidalgo M, Rosa A, Orgilés $\mathrm{M}$. Cuestionarios, inventarios y escalas de ansiedad social para adolescentes: una revisión crítica. Rev Psicopatol Psicol Clin. 2003. 8(1):1-21.

18. Olivares I, Ruiz J, Hidalgo M, García-López L, Rosa A, Piqueras J. Social anxiety scale for adolescents (SAS-A): psychometric properties in a Spanish-speaking population. Int J Clin Health Psychol. 2005;5(1):85-97.

19. García-Campayo J, Sanz C, Pérez-Echeverría M, Campos R, Lobo A. Screening of somatization disorder: validation of the Spanish version of the Othmer and DeSouza test. Acta Psychiatr Scand. 1996;94:411-5.

20. Holloway KL, Zerbe KJ. Simplified approach to somatization disorder: when less may prove to be more. Postgrad Med. 2000;108(6): 89-95.

21. Muñoz F. El estrés académico. Problemas y soluciones desde una perspectiva psicosocial. Huelva: Universidad de Huelva; 2003.

Manuscrito recibido el 1 de agosto de 2007. Aceptado para publicación, tras revisión, el 8 de julio de 2008.
ABSTRACT

The relationship among depression, anxiety, and somatic symptoms in a sample of university students in northern Mexico
Objective. To determine how anxiety, depression, and somatic symptoms are related in a sample of university students in northern Mexico.

Methods. An exploratory study was conducted through self-administered questionnaires applied to a convenience sample of 506 psychology students at two universities in Monterrey, in the state of Nuevo León, Mexico. To evaluate somatic symptoms, the Patient Health Questionnaire was used; for depression, the Beck Depression Inventory; and for anxiety, the Social Anxiety Scale for Adolescents. Spearman's correlation was used to determine to what extent the associations among the variables were significant. The Kruskal-Wallis test was used to compare anxiety and depression levels between groups of students organized by severity of somatic symptoms.

Results. Of the participants, $129(25.5 \%)$ presented somatic symptoms that were of medium intensity or severe; just $4(0.8 \%)$ had severe depression; and only $2(0.4 \%)$ students presented anxiety levels over $75 \%$ of the scale maximum. The severity of somatic symptoms increased in step with anxiety and depression levels. The somatic symptoms occurring most frequently and of greatest concern among the study sample were: headache, menstrual pain, and backache, as well as feeling tired and having difficulty sleeping. Conclusions. The direct association between the severity of somatic symptoms and depression and anxiety was confirmed. It is recommended that all treatment and/or prevention programs addressing one of these conditions, include the other two as well. Programs specifically aimed at university youth should be implemented.

Key words Anxiety, depression, psychosomatic disorders, students, Mexico. 